\title{
A simple test to determine the settling behaviour of slurries in piston diaphragm pumps
}

\author{
H Krimpenfort FELUWA Pumpen GmbH, Germany \\ D Nägel FELUWA Pumpen GmbH, Germany
}

\begin{abstract}
One of the most critical issues when designing a slurry system is sedimentation of solid particles in pipelines and pumps. In pipelines, sedimentation will occur when the transportation velocity of a slurry through the pipe is below the deposition velocity. As soon as particles are settled, there is a considerable risk that, in time, a plug will be created which will block the pipeline completely.
\end{abstract}

Settling of solids can, however, also be detrimental for piston diaphragm pumps; large and heavy solids may settle within the diaphragm housing, on top of the suction valves. This layer of solids will cause diaphragms to rupture. In addition, there is a risk that the settling particles may block the valve, causing pressure surges and further damage to the downstream equipment.

In order to determine the settling behaviour of a slurry, costly and time-consuming loop tests need to be executed in specialised laboratories for which large quantities of solids are required. This test may also be an indication of the settling behaviour of solids within a piston diaphragm pump, but would not be very accurate, leaving risk of damage to diaphragms.

Therefore, a simple, quick and inexpensive test (SE: sedimentation detection system with evaluation algorithm) was developed which indicates if a slurry is prone to settling or not, by determining how fast solids will settle within a diaphragm housing. For this test, just a few kilograms of solids are required, and the result of the test is known within a very short period of time.

In principle, the test is based on the measurement of the speed at which particles settle on a scale; the faster the particles settle, the higher the risk of a settled pipeline and the higher the transportation velocity needs to be.

This paper will describe the methodology of this test, its results and interpretation. Also, a technology will be presented which prevents settling of solids within the diaphragm housing of piston diaphragm pumps.

Keywords: pump systems, sedimentation, test, down-flow

\section{Introduction}

Settling of solids within the diaphragm housing of a piston diaphragm pump can have grave consequences for the operation and availability of such pumps. The layer of settled solids will cause the diaphragm to rupture which will (with single diaphragms) lead to contamination of the propelling liquid area. Contaminated propelling liquid will cause excessive wear to pistons and cylinder liners with loss of pressure as consequence. In order to prevent this, the pump needs to be stopped and the propelling liquid should be replaced with clean oil, resulting in extended shutdown of the pump and reduced availability. If a costly standby pump is not available, the pump's out-of-service condition will result in reduced production capacity or even loss of a plant component.

The SE (sedimentation detection system with evaluation algorithm) test will indicate if the risk of settling of solids is realistic or not. In case the slurry is prone to settling, the direction of the flow through the pump can be adjusted from traditional up-flow to down-flow in which settling of solids will be prevented. 


\section{Currently available methods to determine critical velocity}

For pipelines, a number of settling tests, assessments, and technics have been available on the market for many years, ranging from very simple and inaccurate to very extensive and more accurate. As mentioned, these tests are specifically designed for slurry behaviour in pipelines and may not be suitable for pumps as the diaphragm has another geometry and its behaviour is different to a cylinder pipe. These tests do, however, give an indication of the risk of settling within a pump and are therefore still of interest.

The simplest method is to use the Wilson deposition velocity nomogram (Figure 1). However, this is only valid for sedimentation in horizontal pipes and is rather insufficient for the consideration of pumps since there is no continuous flow. In addition, it is only valid for water-sand mixtures in which the sand grains have a round shape. Other solids differ in behaviour.

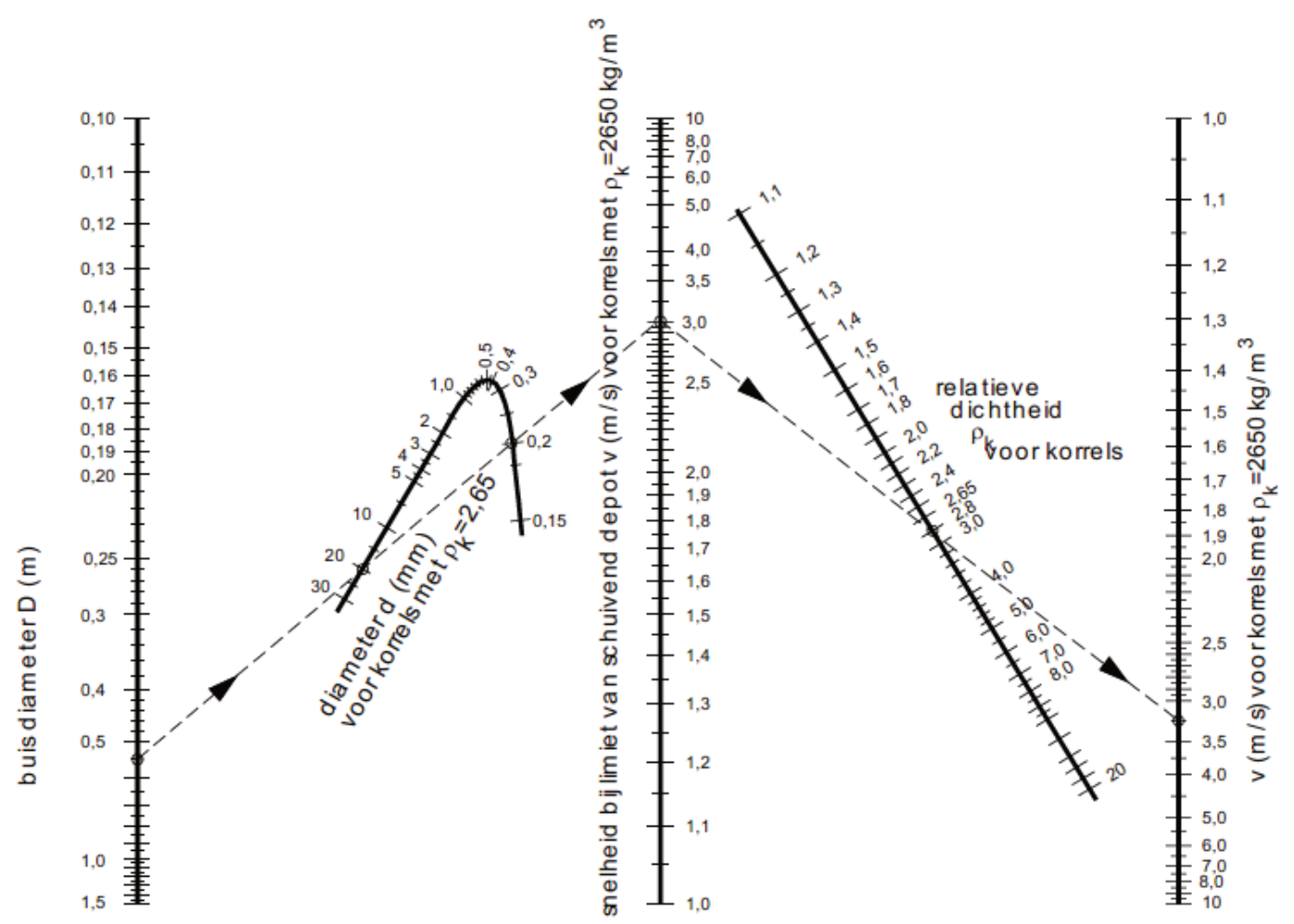

Figure 1 Wilson nomogram - determination of the critical speed (Source: TU Delft OpenCourseWare)

By connecting applicable points (pipeline diameter, particle diameter, specific gravity) on the nomogram the transport velocity for different particle sizes in any size pipeline can be determined.

The other extreme is a full pipeline loop test. These tests are carried out in specialised laboratories of which there are not a lot around in the world. Labs are equipped with several long, closed pipe-loops of different diameters using large centrifugal type pumps as prime movers of the slurry.

The advantage of a loop test is that, in addition to determining the settling velocity of a slurry, also pipeline friction gradients, slurry rheology, pipeline wear, the effect of inclined, vertical, and open channel flow, pipeline start-up and shutdown, and other operational characteristics can be investigated.

These tests, however, are expensive, take a long time (preparation, testing, interpretation, and reporting) and vast quantities of water and solids are required. Since the particles change during the test due to the continuous circulation (especially in the case of softer particles), they must be replaced regularly during the tests, which means additional time and costs. The results, however, are quite accurate, reliable, and diverse.

Figure 2 shows a typical loop test at a laboratory in South Africa. 


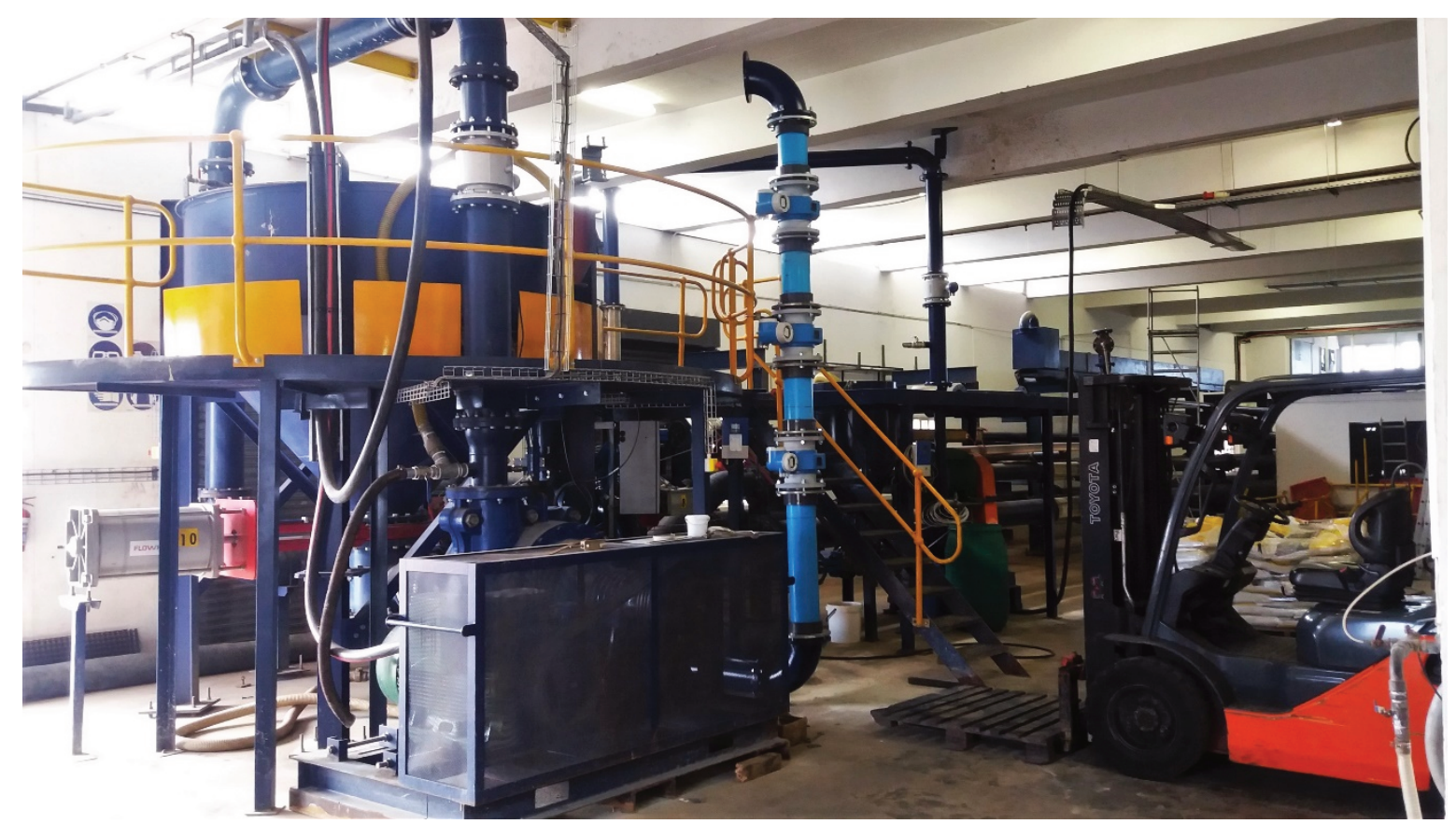

Figure 2 Laboratory loop test (courtesy of Paterson \& Cooke)

Alternative methods are optical, laser, based on weight, measurement of settling zones or other parameters but these all require more time and are more complicated to carry out.

As mentioned, the results of the tests described above give only an indication of the slurry behaviour in a pump. The SE test was specifically developed to investigate the most suitable flow direction in a piston diaphragm pump, either up-flow with potential risk of sedimentation within the diaphragm housing, or down-flow, which avoids the risk of sedimentation in a vertical way.

\section{Description of SE test}

For FELUWA, the background for the development of this test is to prevent settling of solids within the diaphragm housing of a piston diaphragm pump. When solids do settle in a diaphragm housing, a layer of solids will be formed on top of the suction check valve. The hydraulic pressure of the piston will force the flexible diaphragm over the hard layer of settled solids which can cause the diaphragm to rupture. In addition, the valve may fail to open completely. This results in pressure surges and can lead to system damage.

In order to prevent this phenomenon, it is very important to know at which speed the solids will start to settle. In addition to settling, there is also the intermediate stage that the concentration of the particles in the vertical increases but remains constant. In this range, the particles move more slowly than the carrier medium (water), but due to the higher concentration, the inflow and outflow of particles are in equilibrium.

In case it can be expected that settling will occur, the flow direction of the slurry needs to be adjusted from the traditional up-flow (suction on the bottom of the pump, discharge on the top) to down-flow (suction on top, discharge at the bottom of the pump).

When analysing the sedimentation risk inside a diaphragm pump, there are several other parameters to be considered in addition to the factors that apply to a horizontal pipeline.

On one hand, the pumped medium does not flow horizontally inside the pump head, but vertically from bottom to top, so that the gravitational force plays an even greater role here.

Secondly, the flow within the pump head is discontinuous. Thus, medium is sucked in during the suction stroke and discharged during the pressure stroke. The flow velocities can only be varied within the permitted diaphragm load. 
For this reason, FELUWA decided to develop a new type of sedimentation detection system (SE system).

Although the principle of the test is not entirely new, the SE test consists of a few relatively simple and inexpensive instruments which are easy to obtain, install, use, and interpret. In addition, a very limited quantity of (dry) solids are required to carry out the test.

The operating principle is based on the settling speed of solids in a transparent cylinder. The main instruments required for the test are a transparent cylinder, a scale, and a circulation pump.

A more detailed description of the measurement process is as follows:

- The solids are mixed with water to the specified concentration.

- Particles are kept in suspension in the cylinder by circulation pump.

- Pump is stopped and particles will start to settle.

- Particles will settle on a dish which is connected to a scale.

- Weight increase on dish is measured and plotted in time.

Figure 3 shows the instruments required for the test; Figure 4 shows the equipment schematic.

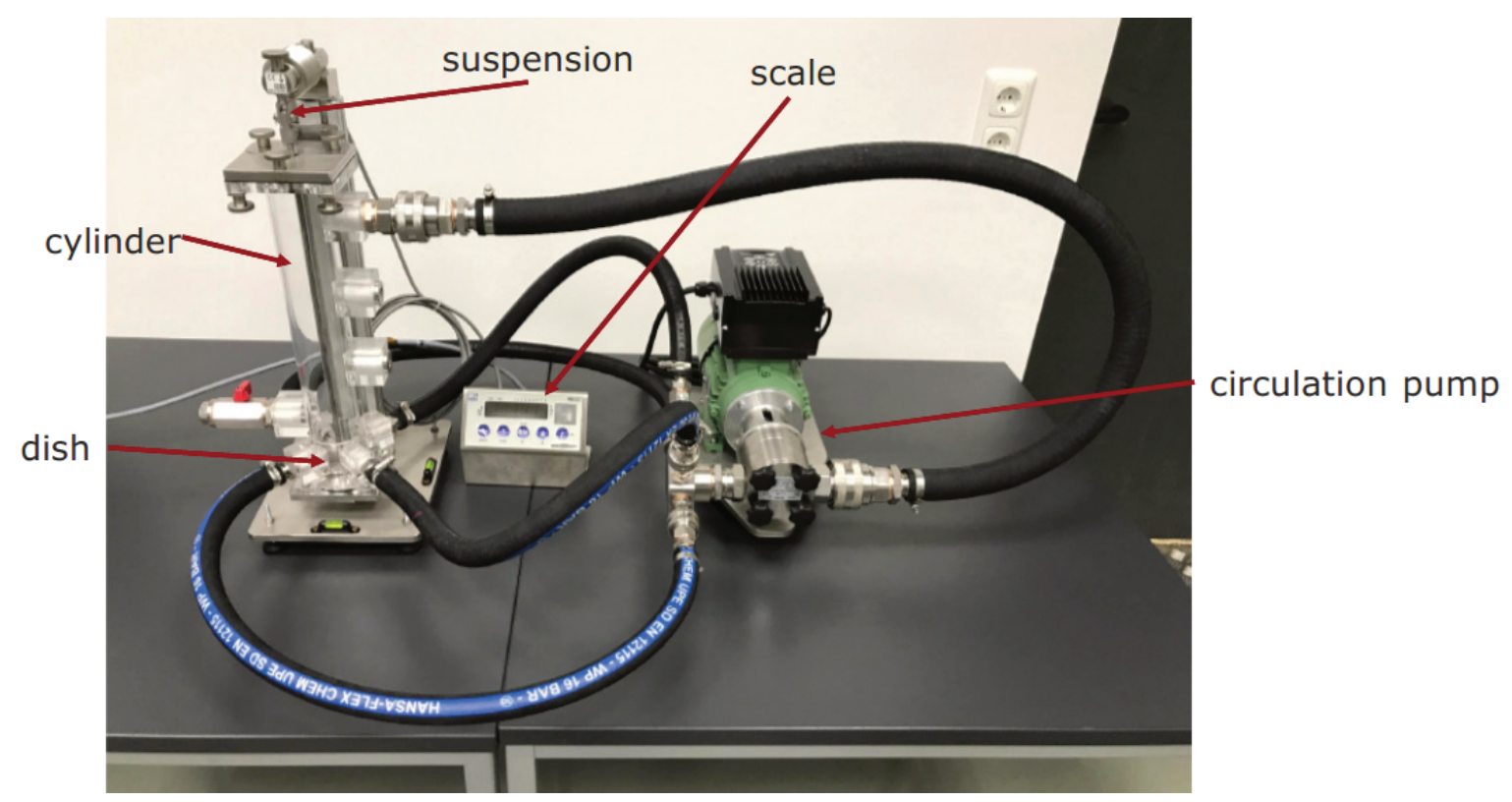

Figure 3 SE test equipment

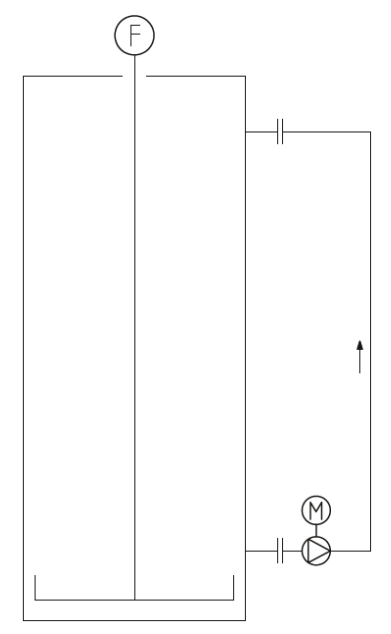

Figure 4 SE test equipment schematic 
The weight increase on the scale is translated into a settling velocity by means of a specially developed evaluation algorithm and indicates if there is a risk of settling of solids within the pump and consequently determining the flow direction through the pump. The test results are known within one day.

In order to provide clients with comprehensive information on the pumpability of the medium and an estimate of the operating costs, in addition to the equipment for the SE test, the laboratory is also equipped with a Miller test machine to determine the abrasivity of slurry particles, a Keyence VHX-6000 digital microscope for particle size distribution, and a rotational viscosity meter.

To test the lifetime of valve components, a triplex pump $\left(20 \mathrm{~m}^{3} / \mathrm{h}\right.$ at $\left.14.000 \mathrm{kPa}\right)$ with pipe-loop is installed in the laboratory.

Figure 5 shows the triplex pump with circulation tank and piping.

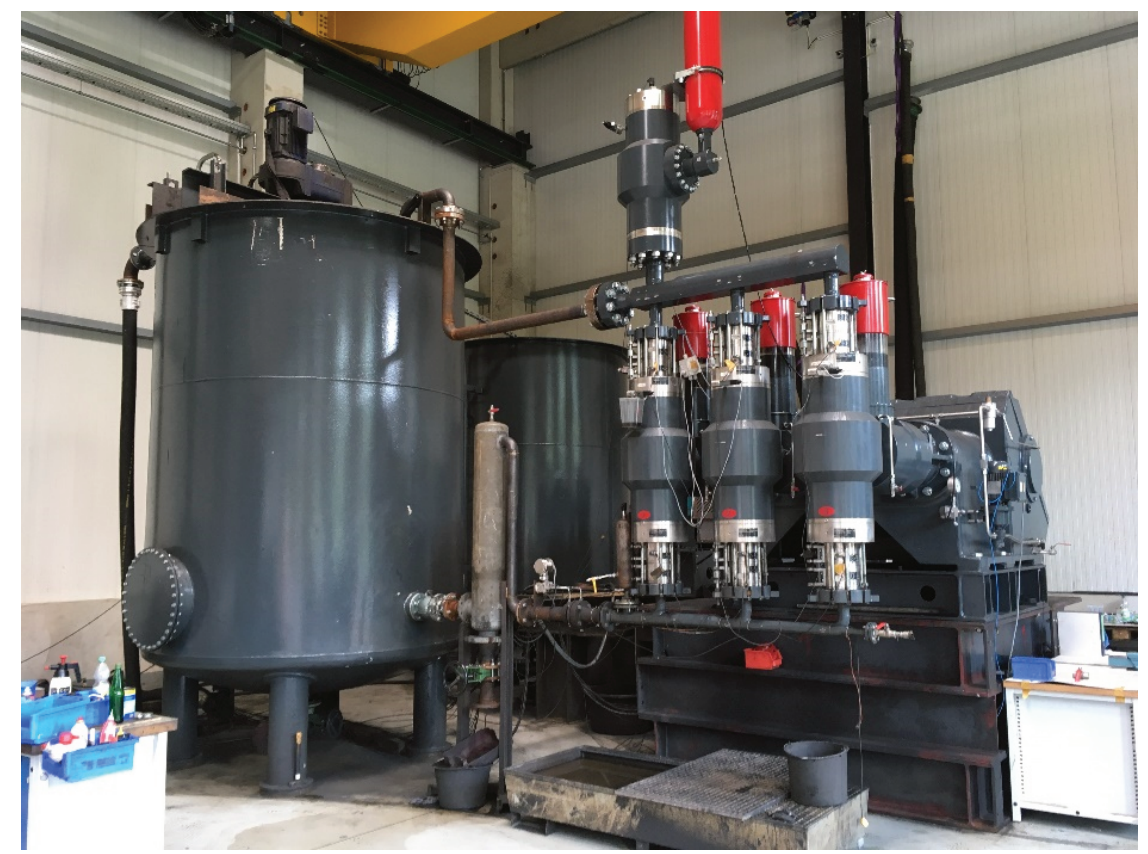

Figure 5 Pump with test loop

\section{Typical results and interpretation}

At the laboratory, many SE tests have been carried out over the years on several types of slurry. The deposition on the dish is measured over time. In homogeneous mixtures, the mass on the dish increases proportionally to time. The more uneven the distribution, the more the increase flattens with time, because the sinking speed of the particles is different.

Therefore, the decisive factors are the slope at the beginning, which measures the particles that sink quickly, and the total time it takes for all the particles to settle.

If there are many rapidly sinking particles, there may be a risk of sedimentation even if the entire deposition process takes a relatively long time. In this respect, sufficient experience has been gathered to decide whether this proportion of rapidly sinking particles is critical or not.

Depending on the flow velocity in the membrane at the lowest number of strokes and the determined critical velocity, the recommendation for the conveying direction is then given. 
The following simplified formula is used to determine the settling behaviour of a slurry:

$$
\Delta s=s_{\text {lift }}-s_{\text {sink }}-s_{\text {safe }}
$$

where:

$$
\begin{aligned}
& \Delta s=\text { sedimentation velocity. } \\
& s_{\text {lift }}=\text { the flow velocity in the diaphragm housing. } \\
& s_{\text {sink }}=\text { the quotient of the hose-diaphragm length and the settlement time. } \\
& s_{\text {safe }}=\text { safety margin (depending on hose-diaphragm length). }
\end{aligned}
$$

\subsection{Interpretation of results}

If the flow velocity is greater than the sum of the sinking and safety speed, the particles per piston stroke rise in the hose-diaphragm and will be discharged.

If the flow velocity is lower, the particles per piston stroke either remain or sink in the pump chamber. The particles begin to settle and will form a layer of solids on the suction valve.

In short:

- If delta $S>0$ = slow settling: up-flow.

- If delta $S<0=$ fast settling: down-flow.

\subsection{Typical examples}

Many slurry samples have been received and tested, two of which are described below (Figure 6). The first example is of an iron ore slurry of which the particle size distribution (PSD) is shown, using the Keyence VHX-6000 digital microscope. From the PSD it can be concluded that the sample contains a few large and many fine particles. The fine particles carry the large ones upward avoiding the risk of settling.

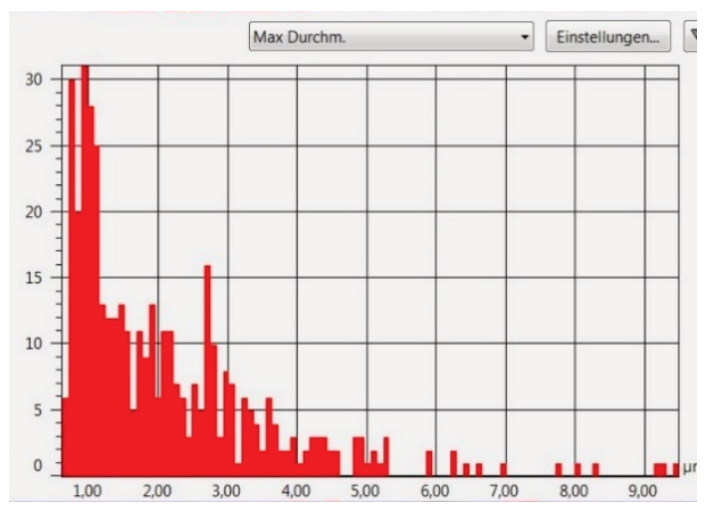

(a)

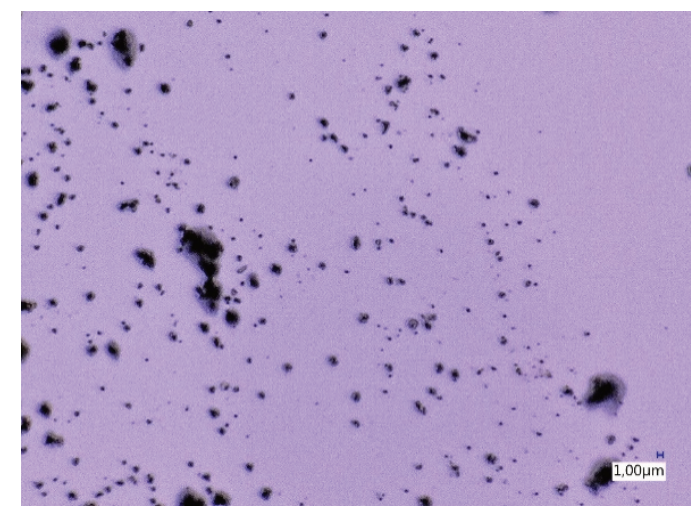

(b)

\section{Figure 6 (a) PSD; (b) Magnified particles of iron ore}

This is confirmed by the SE test. It takes 75 to 80 seconds for all particles to settle on the scale (Figure 7).

After applying the safety margin, the delta $\mathrm{S}$ is higher than zero and a piston diaphragm pump in up-flow configuration can be used for the transfer of this slurry. 


\section{Iron ore}

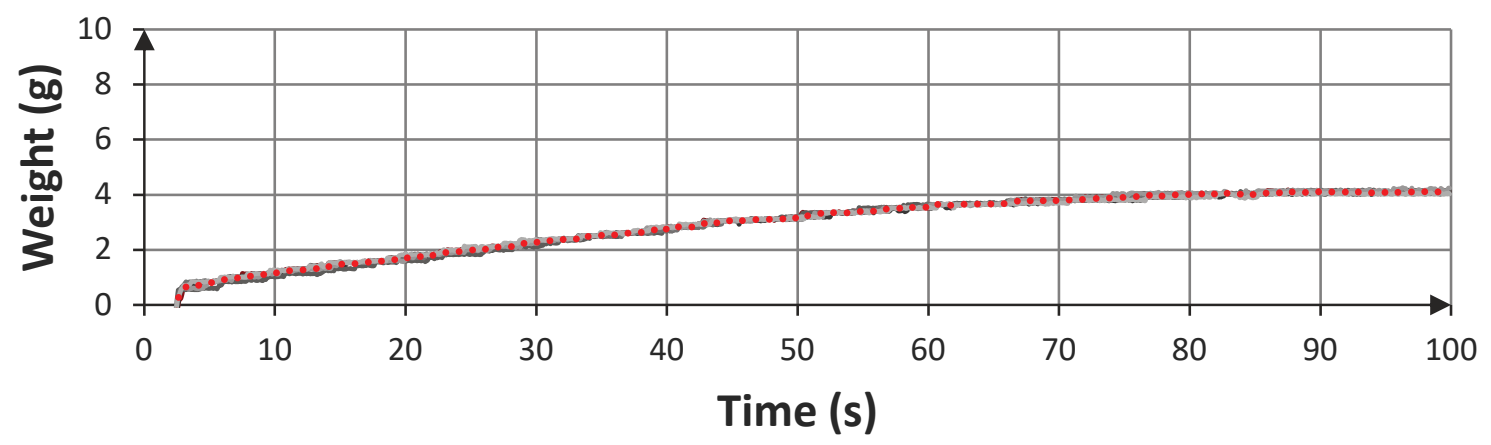

Figure 7 Sedimentation behaviour of a slow settling slurry

Figure 8 shows the PSD and particles of a sand sample.

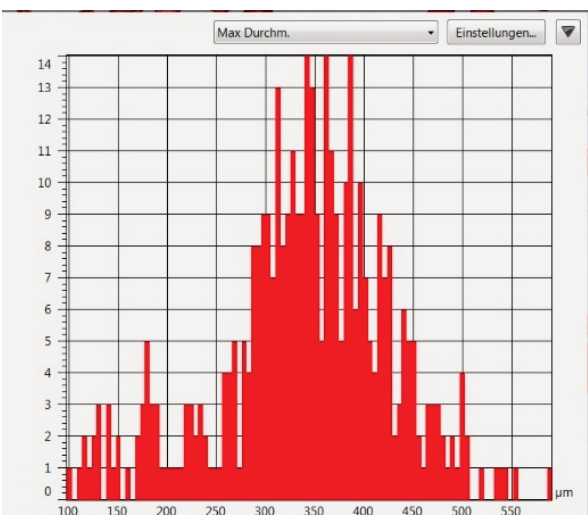

(a)

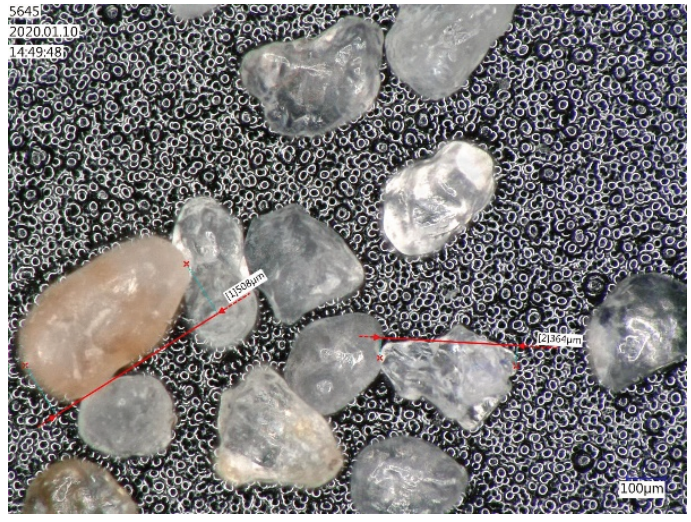

(b)

Figure 8 (a) PSD; (b) Magnified particles of sand

The second example is a sand slurry for a project in the United States of America. As the PSD is heterogenous and the sand particles are rather round, it was concluded that the particles are prone to settling. Figure 9 shows a result of an SE test on the sand slurry. In this case, it takes nine seconds for all particles to settle on the scale.

\section{Sand}

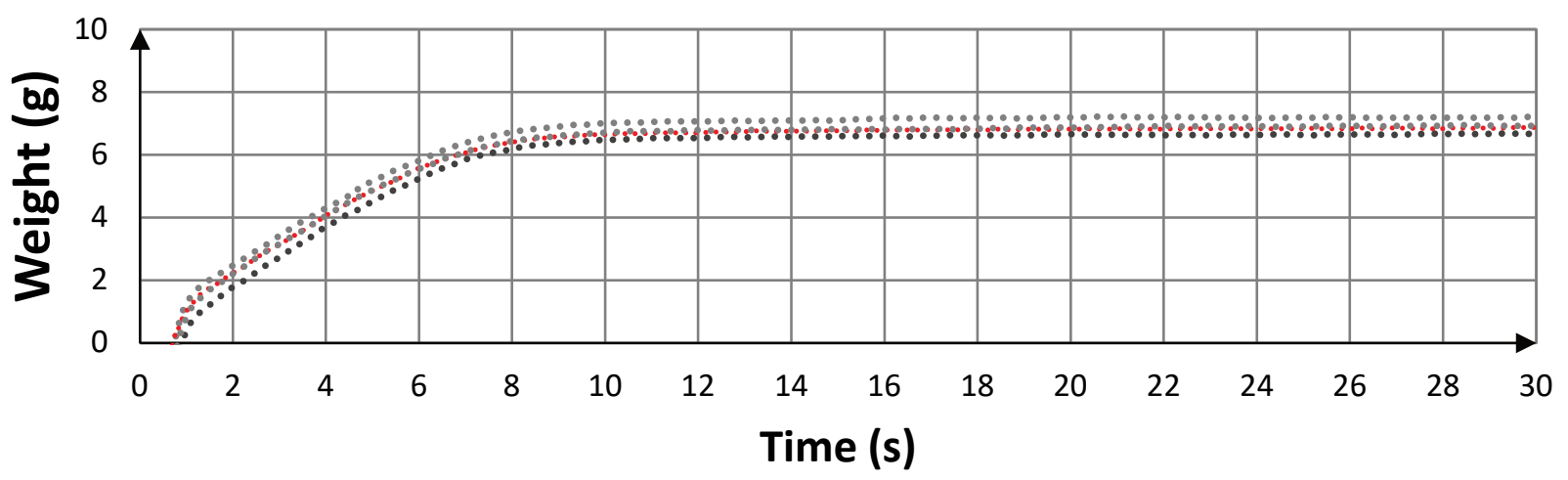

Figure 9 Sedimentation behaviour of a fast-settling slurry 
After applying the safety margin, the delta $S$ is lower than 0 . Consequently, a piston diaphragm pump in down-flow configuration is required for the transfer of this slurry.

For critical applications, customers are invited to send samples of the solids that need to be pumped to the laboratory for testing.

\section{Down-flow technology}

In case there is a risk of slurry particles settling within the diaphragm housing, even at higher stroke rates, the only solution to prevent settling of these solids on the suction valves (and subsequent diaphragm damage as a result) is to reverse the flow direction through the pump from upward to downward (Figure 10).

Here, the slurry test facility also offers the possibility to carry out a test with real slurry in order to examine critical media in a realistic test setup to analyse whether the medium can still be pumped safely by means of up-flow or if a down-flow configuration is a must.

The risk of settling of solids with homogenous slurries is limited as small particles present in the slurry will carry the larger and heavier particles through the pump against gravity.

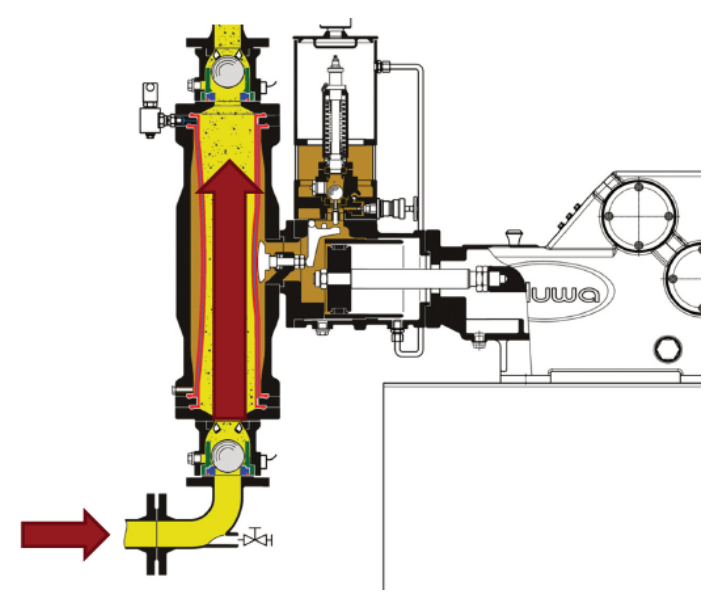

Figure 10 Cross-section of a piston diaphragm pump in up-flow configuration

In heterogenous slurries, however, with a limited number of small particles to carry larger particles, this risk is far greater. Although during the discharge stroke, these large particles will be transferred upward by a certain distance, and gravity will pull these particles downward over a greater distance (Figure 11).

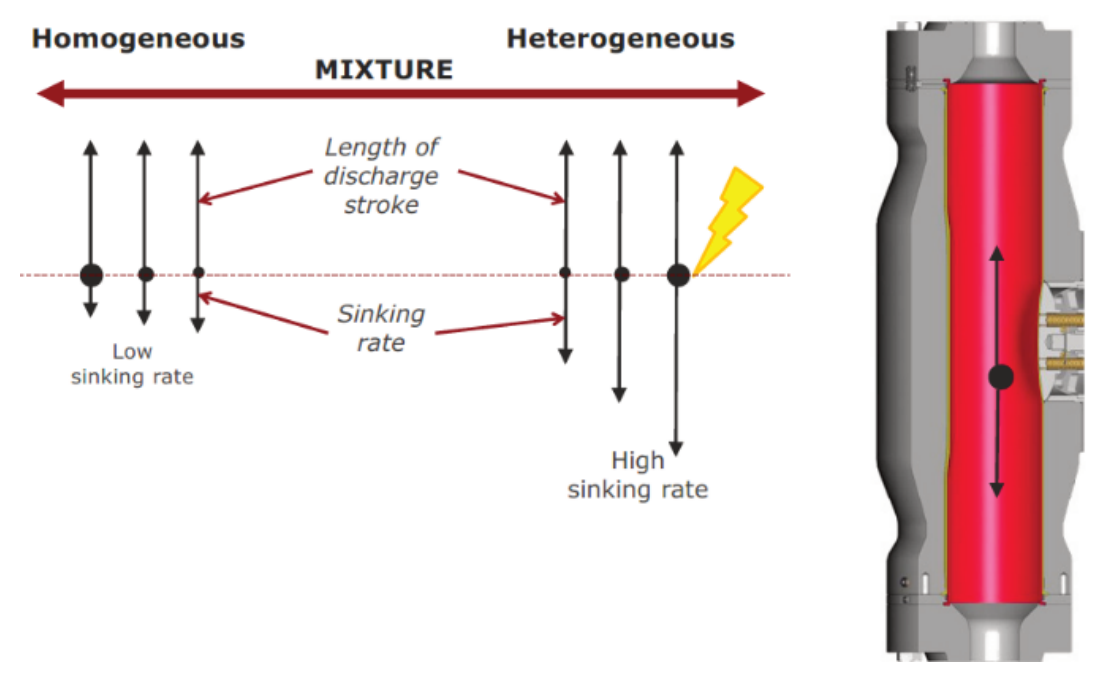

Figure 11 Settling velocity of course solids against gravity 
In time, these large particles will accumulate on the suction valves, hindering these valves from opening and building a layer of material.

The hydraulic force of the pistons will push the flexible diaphragm over the layer of settled solids into the discharge valve and cause the diaphragms to rupture (Figure 12). As a result, the propelling liquid (unless the pump is equipped with double diaphragms) will be contaminated with consequent long pump interruption due to the required cleaning of the propelling liquid area.

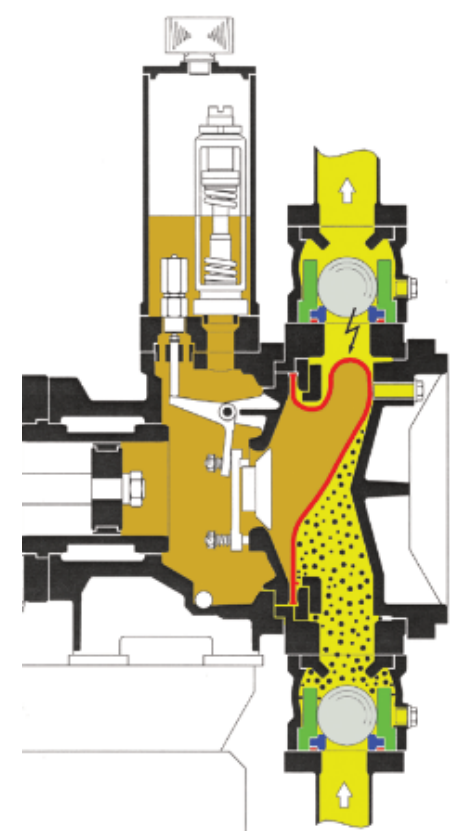

Figure 12 Diaphragms are forced into discharge valves by hydraulic pressure

The risk of diaphragm damage can easily be prevented by reversing the direction of the flow through the pump from upward to downward (Figure 13). When pumping downward, gravity assists the pump in evacuating large and heavy solid particles through the pump.

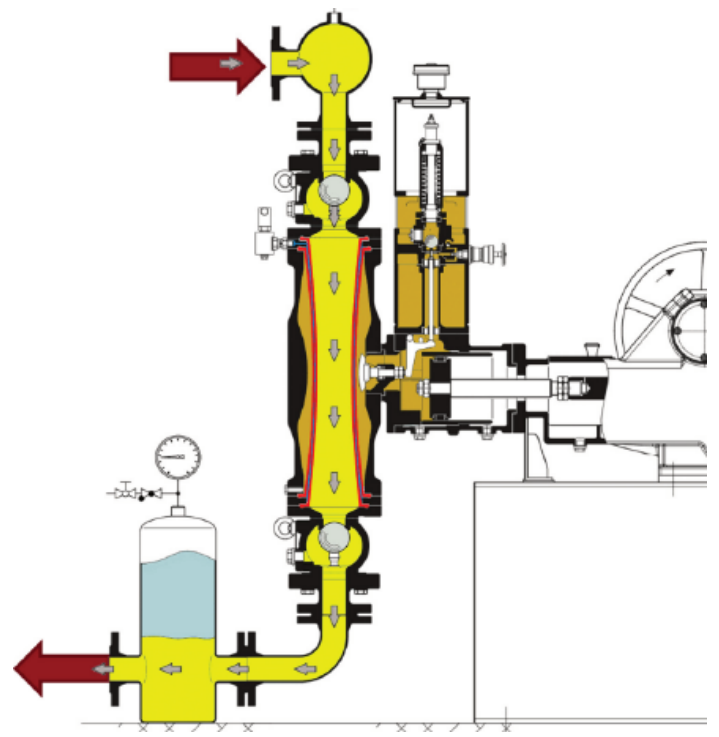

Figure 13 Piston diaphragm pump in down-flow configuration with slurry flowing from top to bottom

Key to this technology are the valves. Rather than closing downward, they must close upward. For this reason, the valve cones or balls are supported by springs or the balls are hollow to make them lighter. 
Pumps equipped with down-flow technology have been installed very successfully for many applications worldwide such as the transfer of fly ash slurry through pipelines, feeding autoclaves with gold slurries, iron ore and copper tailings, etc.

One example that may be of particular interest is the Boleo tailings transfer project where four huge pumps with down-flow technology (Figure 14) have been in operation for eight years. These are five-cylinder single-acting pumps which have a capacity of $750 \mathrm{~m}^{3} / \mathrm{h}$ each at a pressure of 45 bar (they are capable of a capacity of $850 \mathrm{~m}^{3} / \mathrm{h}$ at a pressure of $85 \mathrm{bar}$ ) and weight 150 metric tonnes each. As the slurry has a solid content of only $28 \%$ (it cannot be thickened any further), there was a risk that the solids would settle within the diaphragms.

In order to avoid this from happening, they are equipped with down-flow technology.

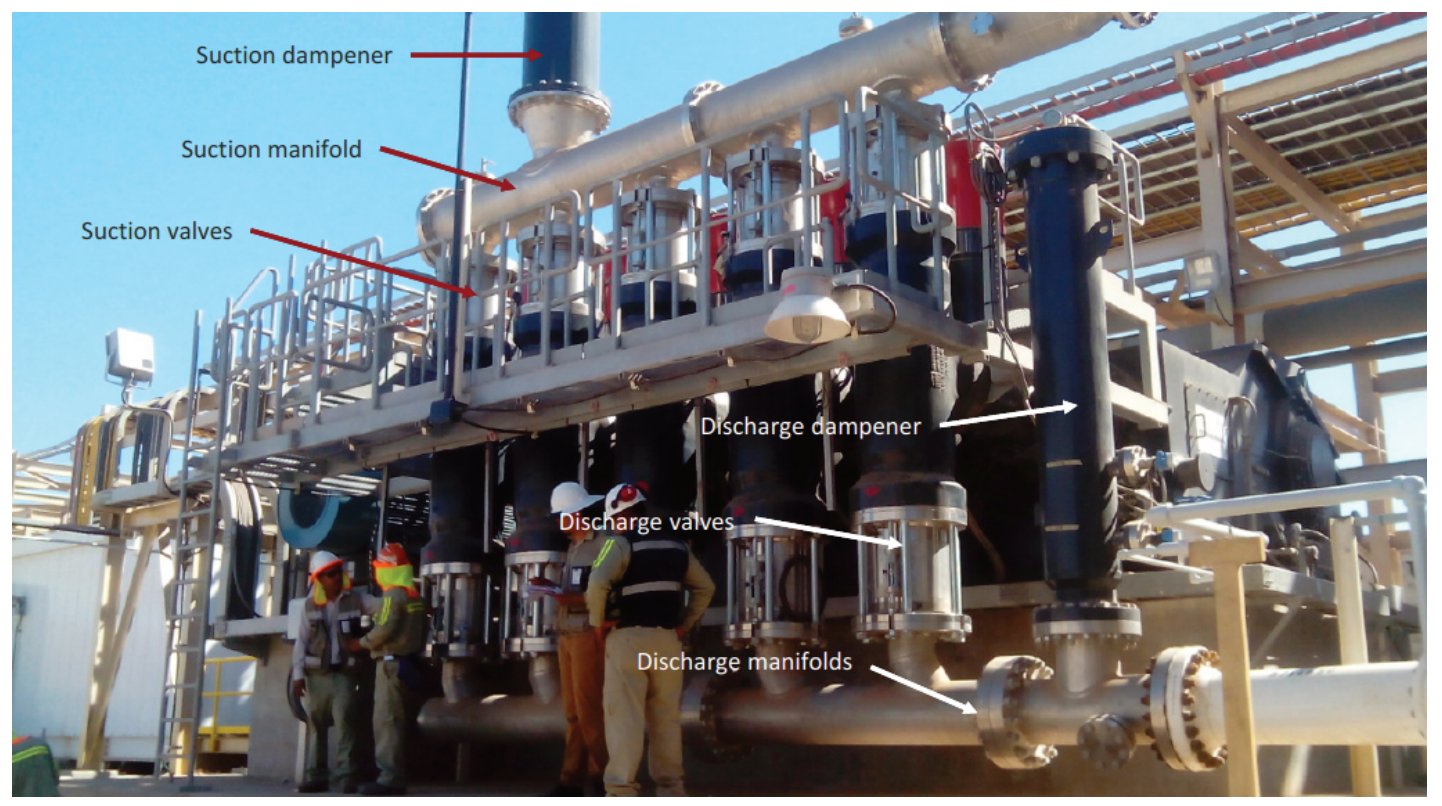

Figure 14 Five-cylinder QGK500-5DS350-DFT pumps

It may be also be of interest to know that up-flow pumps can be retrofitted to down-flow within a matter of days by replacing the position of the suction and discharge piping, dampeners and the internal parts of the valve cassettes.

\section{Summary and conclusion}

Sedimentation is one of the main causes of early failure of diaphragm pumps. By carrying out the SE test, the risk of sedimentation inside the pump head at a very early stage of the project can be detected and prevented, avoiding costly and time-consuming adjustments to the pump and system at a later stage.

The SE test gives a clear indication as to whether a traditional up-flow diaphragm pump can be used or not. In case the sedimentation is within the critical range (delta $S$ below zero, see Section 4), it is recommended to apply down-flow technology.

In order to avoid sedimentation, the double hose-diaphragm pump has the ability to turn the traditional pumping principle on its head (i.e. pumping takes place from top to bottom). Furthermore, the cylindrical shape of the diaphragm and straight flow path prevents solids from settling in dead pockets.

Liquids with large solids also require special, tailor-made conveying valves. For reversal technology, various versions are available that allow coarse solids in the feed stream, such as spring-loaded ball valves, ball spherical valves or special floating ball valves. In processes where continuous conveying must not be interrupted, double valves in cassette design prevent backflow leakage as a result of trapped solids.

Down-flow technology has been applied on many slurry transfer pumps and is proven technology. 